\title{
Perspectivas Metodológicas na Pesquisa Sobre o Comportamento do Consumidor
}

Methodological Perspectives on Consumer Research

Perspectivas Metodológicas em la Investigación Sobre el Comportamiento del Consumidor
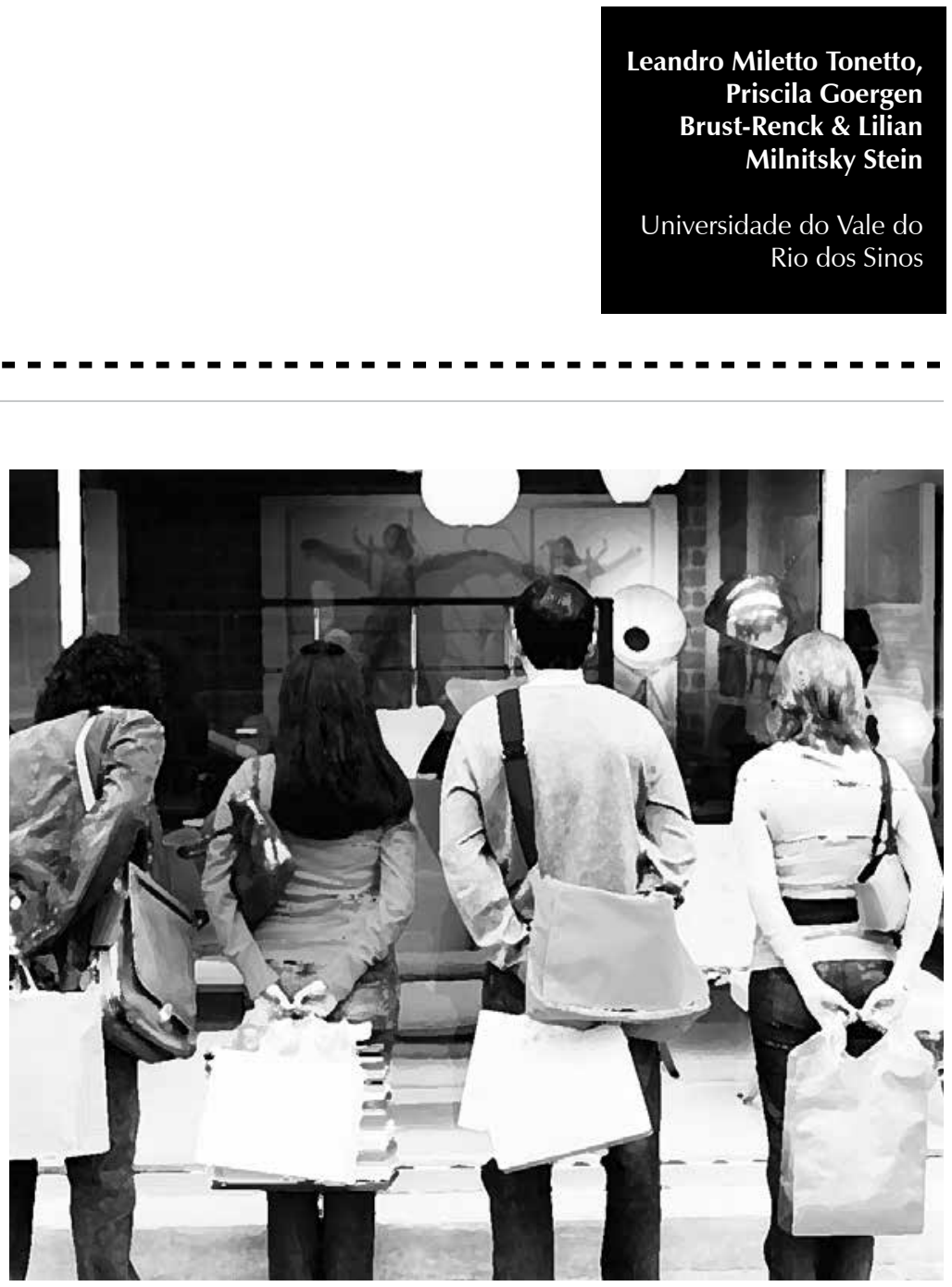
Resumo: Apresenta-se neste artigo uma revisão crítica da literatura sobre métodos de pesquisa em Psicologia com uma orientação para estudos de consumo, acompanhada de exemplos da prática profissional dos autores junto a empresas que investigam o comportamento dos consumidores. A realidade de mercado exige que o pesquisador saiba escolher e empregar o método mais apropriado para o problema de pesquisa (exploratório, descritivo, experimental), o que institui a necessidade de classificar, descrever e comparar os diferentes métodos. A contínua transformação do consumidor e a constante evolução do mercado também tornam necessária a integração desses métodos em uma ferramenta essencial que oportunize a compreensão dos fenômenos em estudo. Conclui-se que o psicólogo deve saber diferenciar o emprego de diferentes métodos de pesquisa frente a demandas específicas de compreensão dos consumidores, em uma orientação que parte da prática profissional e não apenas de orientações metodológicas pré-estabelecidas com base na teoria.

Palavras-chave: Comportamento do consumidor. Psicologia do consumidor. Consumo. Economia Comportamental.

Abstract: The present paper presents a critical review of the literature about research methods in psychology with a focus on consumer studies, followed by examples of the professional practice of the authors in consumer behavior research. The market reality urges the researcher to be able to choose and make use of the most appropriate method to solve the research problem (exploratory, descriptive, experimental), what creates the need to classify, describe and compare the different methods. The scenery and the consumer in constant transformation also brought the call for an integrative view of the different methods into an essential tool to the comprehension of the study phenomenon. The conclusion is that the psychologist should know how to differentiate the need to use each distinct research method when facing specific requirements to understand consumers based not only on pre-established methodological rules from theory, but also based on guidance from professional practice.

Keywords: Consumer behavior. Consumer psychology. Consumption. Behavioral economics.

Resumen: En este artículo se presenta una revisión crítica de literatura sobre los métodos en investigación psicológica orientados para los estudios de consumo, acompañado por ejemplos de la práctica profesional de los autores en empresas con investigaciones sobre el comportamiento de los consumidores. El escenario actual del mercado exige por parte del investigador un conocimiento del método más eficaz para el problema de investigación (exploratorio, descriptivo, experimental), que abarca la necesidad de clasificar, describir y comparar los diferentes métodos. La constante transformación del escenario y del consumidor generó una necesidad de integrar esos métodos como herramientas esenciales. En conclusión el psicólogo deberá saber diferenciar la necesidad de implementar diferentes métodos de investigación para las empresas, debido a las demandas específicas de los consumidores orientados a partir de la práctica profesional y no solo en función de las orientaciones metodológicas preestablecidas en función de la teoría.

Palabras-clave: Comportamiento del consumidor. Psicología del consumidor. Consumo. Economia del comportamiento.

O cenário do mercado contemporâneo é marcado por mudanças ambientais drásticas, pelo crescimento não ordenado das empresas e pela inconstância na sua sustentabilidade. A capacidade produtiva excedeu a demanda de consumo, o que fez com que o comportamento do consumidor e suas motivações mudassem. O consumidor passou, então, a ser mais importante e a exigir mais retorno por seu dinheiro, o que levou ao aumento da concentração de forças no que agrega valor a determinado produto ou serviço e, ao mesmo tempo, à eliminação do que não agrega (Malhotra \& Peterson, 2001; Hassan, Craft, \& Kortam, 2003).

Para a compreensão desses fenômenos, o campo de investigação das ciências do consumidor constituiu-se de forma interdisciplinar, com acentuada participação da Psicologia (Erasmus, Boshoff, \& Rousseau, 2001; Foxall, 2001, 2003; Simonson, Carmon, Dhar, Drolet, \& Nowlis, 2001). Nesse contexto, o trabalho do psicólogo em estudos de consumo para empresas tornouse fundamental. Mediando relações entre as demandas internas do consumidor e as empresas, o profissional de Psicologia passou a enfrentar desafios profissionais diversos frente ao novo mercado que se abria.

Segundo Simonson et al., embora a Psicologia tenha causado o maior impacto na pesquisa sobre o comportamento do consumidor, outras disciplinas também tiveram importantes influências. Os métodos de pesquisa acompanham essas tendências, uma vez que se originam de diferentes áreas. 
A oposição entre abordagens interpretativas ou pós-modernas (que visam a uma interpretação subjetiva dos dados) e positivistas (que visam a investigar relações do tipo causa-efeito), por exemplo, tendem a causar divergências nos tipos de métodos a serem utilizados.

Assim como há uma diversidade de aportes teóricos, existe, da mesma forma, uma gama de possíveis métodos a serem utilizados na pesquisa sobre o comportamento do consumidor que são adequados para buscar respostas a problemas diferenciados. O psicólogo, profissional cuja formação tem uma importante interface com a teoria, muitas vezes associa métodos de pesquisa à orientação teórica, como, por exemplo, a abordagem experimental dos estudos cognitivos ou o caráter exploratório de muitos dos estudos da Psicologia social. No entanto, em trabalhos orientados para o mercado, a escolha do método mais eficaz para responder ao problema de pesquisa desejado é uma imposição do problema em si, de orientação prática. O objetivo deste artigo, nessa direção, é classificar e descrever os métodos de pesquisa (exploratório, descritivo e experimental), demonstrando suas indicações de acordo com a orientação para o mercado. Acredita-se, dessa forma, que exista uma importante contribuição para o psicólogo, na medida em que traz uma nova perspectiva sobre um campo já dominado por esse profissional - a pesquisa -, porém com outra perspectiva - a de orientação para o mercado consumidor.

Na busca de resposta sobre a adequação de diferentes métodos para cada tipo de problema de pesquisa sobre o comportamento do consumidor, são exploradas, a seguir, as indicações para cada um dos três tipos de pesquisa (exploratória, descritiva e experimental), destacando-se semelhanças e diferenças e priorizando-se uma visão comparativa e integradora, conforme se pode observar na Tabela 1. Cada seção é ilustrada com exemplos de pesquisas, priorizando-se aqueles de interesse para a atuação do psicólogo na área.

Tabela 1. Características dos tipos de pesquisa

\begin{tabular}{llll}
\hline & Exploratória & Descritiva & Experimental \\
\hline Tipos de dados ou & Informações usualmente qualita- & Dados usualmente quantitativos, & Dados essencialmente quantitativos \\
informações & tivas, mas há a possibilidade de & mas com a alternativa de uso de & \\
& outros tipos de dados. & informações qualitativas &
\end{tabular}

\section{Influências do pes- $\quad$ mente com o fenômeno direta- quisador na coleta e dado, modificando-o.}

nos resultados

Noção de amostra e Estudos essencialmente qualitarepresentatividade tivos não têm preocupação com generalização de resultados para além da população estudada.

\begin{tabular}{ll}
\hline Entendimento & Preocupação com o entendimen- \\
do problema x & to sobre a natureza do problema \\
predição de com- & estudado, sobre como se dá o \\
portamentos & fenômeno.
\end{tabular}

Tendências (uso de internet e métodos informatizados)

\section{A internet e os métodos informa- tizados surgem não apenas para a coleta de dados e informações mas também para a realização de análises.}

Pontos fortes
Explora um novo espaço de conhecimento, sem a pretensão de apresentar dados conclusivos.

\section{O pesquisador evita interferir na realidade estudada.}

Dados quantitativos necessitam de amostra representativa para a população estudada.

Preocupa-se com a predição de comportamentos. Os dados são úteis quando servem como base para a tomada de decisão segura.

A internet e os métodos informatizados surgem não apenas para a coleta de dados e informações mas também para a realização de análises.

Visa a descrever ou a mapear uma realidade de mercado, oferecendo um retrato fiel dessa realidade.
O pesquisador não interfere nos experimentos, mas manipula variáveis, modificando a realidade de dada amostra.

Dados quantitativos necessitam de amostra representativa para a população estudada.

Preocupa-se com a predição de comportamentos. Os dados são úteis quando servem como base para a tomada de decisão segura.

A internet e os métodos informatizados surgem não apenas para a coleta de dados e informações mas também para a realização de análises.

Seu ideal é a apresentação de dados conclusivos sobre um problema, estabelecendo relações de causa e efeito. 


\section{A pesquisa exploratória no estudo do comportamento do consumidor}

Pesquisas exploratórias são utilizadas quando se deseja obter dados sobre a natureza de um problema. Utiliza-se esse tipo de pesquisa quando não há informações estruturadas o suficiente para que seja possível conduzir uma pesquisa descritiva ou experimental, ou quando o interesse do projeto é justamente obter um volume de informações que explorem em profundidade como dado fenômeno ocorre (Malhotra, 2006).

Livramento Hor-Meyll e Pessôa (2013), por exemplo, investigaram valores individuais que motivam mulheres de baixa renda na compra de produtos de beleza. Foram realizadas entrevistas em profundidade com 17 mulheres (descritas no estudo como de baixa renda) residentes no Rio de Janeiro. Como é comum em estudos exploratórios, uma grande variedade de resultados foi apontada. Entre os resultados, destaca-se que as consumidoras pareceram utilizar tais produtos na busca de elevação da autoestima e do respeito de classes ditas superiores. Determinadas marcas, ainda, surgiram como referências de qualidade, mas não como fontes de obtenção de status.

A pesquisa exploratória geralmente utiliza informações qualitativas, mas, apesar disso, não deve ser entendida unicamente como pesquisa qualitativa. As informações qualitativas são, geralmente, as mais volumosas desse tipo de pesquisa, mas dados de Censo e de outras fontes secundárias, por exemplo, podem vir a integrá-las, como na necessidade de caracterização de um grupo de consumo (Malhotra \& Peterson, 2001; Vieira \& Tibola, 2005).

A coleta de informações em pesquisas exploratórias ocorre, em estudos de consumo, muito comumente em uma perspectiva etnográfica, por meio de observações participante e não participante, de entrevistas informais e casuais, de diários de informações e da inserção do pesquisador no ambiente natural dos consumidores. A utilização dessas técnicas usualmente tem longa duração, podendo chegar a meses de pesquisa. $\mathrm{O}$ uso desses procedimentos visa a permitir que o fenômeno observado seja conhecido com espontaneidade e vivido como um incidente, pois o pesquisador é o instrumentochave para a coleta do material de pesquisa e sua análise. Nessa direção, empresas como a Hewlett-Packard, IBM, Motorola, Xerox e McDonald's, dentre outras, têm utilizado pesquisas para entender como os consumidores usam seus produtos, valendo-se de pesquisas exploratórias (Canniford, 2005; Coupland, 2005).

Os participantes são integrados ao estudo em pequenos grupos e de forma intencional, já que, dessa maneira, pode-se selecionar pessoas que forneçam informações pertinentes aos objetivos investigados (Canniford, 2005; Coupland, 2005). Nesse caso, o termo amostra representativa torna-se inadequado, pois é oriundo da Estatística e tem implícita a noção de que os participantes da pesquisa são representativos de uma população maior e de que as informações discutidas no relatório de pesquisa podem ser generalizadas. A representatividade do que é discutido em uma pesquisa exploratória, contudo, é restrita ao universo pesquisado, pois seus objetivos envolvem o entendimento dos processos de como ocorre um fenômeno, e não sua predição.

No entanto, a interação entre pesquisados e pesquisadores, como nos casos de estudos de inspiração etnográfica, não é a única tendência no que se refere ao uso de técnicas de coleta de informações. Entre as fontes de dados em pesquisas exploratórias, a internet aparece como importante fonte de dados. 
Os grupos focais (técnica na qual um grupo de consumidores interage discutindo tópicos propostos pelo pesquisador), por exemplo, são citados como extremamente práticos quando realizados pela internet, já que a transcrição do material é instantânea, e, além disso, pessoas de diversas partes do mundo podem interagir com facilidade (O'Connor \& Madge, 2003). Ainda, os métodos informatizados surgiram não apenas como alternativas para a coleta de informações mas também para sua análise, por meio de softwares específicos para análises qualitativas.

As inovações tecnológicas encontram-se em expansão nos estudos exploratórios sobre o comportamento do consumidor. Barboza e Arruda Filho (2012), a fim de compreender o consumo tecnológico com apelo ecológico, por exemplo, utilizaram, em pesquisa exploratória, a netnografia, ratificando as tendências de uso da internet. Os autores extraíram informações de blogs e de outros sítios para análise qualitativa.

A pesquisa exploratória pode alcançar elementos de dada realidade que outros métodos não atingem (ver Tabela 1). Pode-se averiguar se os consumidores se comportam como dizem ou pensam. Esse tipo de informação pode trazer esclarecimentos mais pertinentes à realidade do consumo, já que são relacionados à forma como os consumidores compram ou utilizam produtos de certa marca. Auxiliam, ainda, a entender simbolismos, significados do consumo, bem como a inserção de produtos e marcas na experiência social e cultural dos consumidores (Torres, Allen, \& Pérez-Nebra, 2006). A pesquisa exploratória possibilita, nessa direção, que sejam confrontadas as intenções dos participantes - que ocorrem no nível dos pensamentos - com o que ocorre de fato (Canniford, 2005; Coupland, 2005; Nepomuceno, Porto, \& Rodrigues, 2006).
Para Malhotra e Peterson, variáveis investigadas de forma exploratória podem ser emoções, metáforas, comunicação não verbal e imaginação visual, por exemplo. Os estudos exploratórios, portanto, não são os mais adequados para mapeamento de realidade, como os descritivos, ou para a busca de inferências causais, como os experimentais, já que se limitam à busca de insights e à promoção de entendimento acerca do contexto no qual dados fenômenos ocorrem (ver Tabela 1 para uma comparação direta).

O processo exploratório não está relacionado à descrição ou à busca de conclusões sobre um problema de pesquisa, mas à compreensão do processo através do qual um fenômeno é produzido. Trata-se, muitas vezes, de um processo de pesquisa mais demorado e de menor potencial de aproveitamento de dados em grandes escalas, mas muito eficaz para buscar compreender mais amplamente os fenômenos em estudo. Muitas vezes, é necessário integrar uma etapa exploratória a projetos que utilizam como método principal uma abordagem descritiva ou experimental, já que a falta de compreensão sobre como ocorre o fenômeno comumente pode impedir a escolha de variáveis dependentes e independentes para um estudo experimental ou até mesmo de alternativas para questões fechadas em questionários de levantamento.

Pesquisas exploratórias são usualmente empregadas quando não se conhecem as respostas possíveis dos consumidores (o que inviabilizaria uma abordagem quantitativa ou descritiva). Gouvêa, Oliveira e Nakagawa (2013), como estratégia para lidar com uma realidade desconhecida, utilizaram pesquisa exploratória com o objetivo de identificar atributos relacionados à lealdade dos clientes a fornecedores e a canais de comercialização. Os autores compararam as respostas dos clientes que compram livros, CDs e DVDs pela internet com as respostas daqueles que compram em loja. 
Em contraste, outros estudos, como o de Fontes, Borelli e Casotti (2012), investigam aspectos de difícil mensuração, como a construção da identidade masculina. O objetivo da pesquisa foi contribuir para compreender o consumidor de produtos e de serviços de beleza, utilizando, para tanto, dez entrevistas em profundidade com homens cariocas. Os resultados, nessas pesquisas, tendem não a ser uma lista de atributos, como no caso supracitado, mas a compreensão de como um fenômeno ocorre. Os autores apontaram, por exemplo, que comportamentos femininos podem servir de parâmetro ou de ponto de referência, a partir dos quais os homens estabelecem o que é permitido ou não em termos de cuidados com a beleza, construindo tal identidade a partir da negação do feminino.

Um exemplo desse fato é o estudo desenvolvido por Oliveira e Gouvêa (2010) com foco na pesquisa relativa a ações de cunho social e na sua relevância para as decisões de compra dos consumidores e sua importância frente a outros fatores. Foi realizada uma pesquisa em duas etapas, sendo a primeira exploratória, a fim de identificar os fatores de influência para a decisão de compra, e a segunda, descritiva, no intuito de avaliar a dimensão da relevância dos fatores apontados no primeiro estágio do estudo. Os dados demonstraram que as ações sociais são menos importantes nas decisões de compra do que outros fatores relacionados no estudo, e que apenas uma reduzida parte da população pesquisada conhece as ações sociais desempenhadas pelas empresas produtoras.

Como é possível perceber, uma diversidade de problemas relacionados ao estudo do comportamento dos consumidores pode ser respondida através dos métodos exploratórios (ver Vieira \& Tibola, 2005, para uma revisão das vantagens e das desvantagens do uso de cada técnica de pesquisa exploratória). Frequentemente, no entanto, os dados necessários necessitam de generalização, exigindo o emprego de outros métodos, conforme se explora a seguir.

\section{Comportamento do consumidor e pesquisa descritiva}

A pesquisa descritiva é usualmente utilizada quando se deseja mapear dada realidade de mercado (Fowler, 1993). Considerando que o objetivo desse tipo de estudo é oferecer um retrato da realidade, os métodos quantitativos são os mais adequados, pois o uso da Estatística visa a assegurar que o mapeamento realizado seja representativo do que se pode observar na população-alvo. Comumente, as empresas utilizam dados de pesquisas descritivas para entender o potencial de mercado para o lançamento de novos produtos, observar o grau de satisfação dos consumidores, conhecer a importância de determinados atributos para uma decisão de compra e conhecer hábitos de compra, conforme se pode observar em avaliações de serviços de ensino superior (Abdullah, 2006).

Secchi, Silva, Del Corso e Tortato (2012) avaliaram percepções de risco em compras pela internet por meio de questionários eletrônicos. O uso da abordagem quantitativa permitiu comparar objetivamente percepções de homens e mulheres em relação a aspectos financeiros, de desempenho, psicológicos, físicos, sociais e de tempo/conveniência de tais compras no que se refere a riscos percebidos. Os autores conseguiram classificar as dimensões por ordem, considerando o sexo dos participantes justamente em função da objetividade do método.

Outras questões de interesse incluem: Como deve ser distribuído tal produto? Qual deverá ser o segmento-alvo para o produto X? Quais as modificações necessárias em dado serviço? 
Fabris, Steiner Neto e

Toaldo (2010) desenvolveram um estudo que utilizou ambas as abordagens referidas exploratória e descritiva - a fim de avaliar, entre universitários, a influência dos agentes de socialização (família, escola, mídia e pares) em relação aos hábitos de reciclagem, ao conhecimento sobre o assunto e aos sentimentos relacionados ao tema.
A pesquisa descritiva trata, nessa direção, de relatar às empresas o que ocorre na realidade (Aaker, Kumar, \& Day, 2004). Como a intenção do pesquisador, ao utilizar tal tipo de método, é alcançar a classificação de problemas, deve-se atentar para os possíveis vieses em relação à seleção da amostra e das técnicas mais adequadas para a coleta de dados (Gilgun, 2005). Um problema adicional na realização de projetos descritivos é justamente a necessidade de obtenção de amostras representativas da população que se pretende investigar para que os dados de uma pesquisa sejam generalizáveis. A representatividade geralmente pode ser alcançada por meio da definição do tamanho da amostra, o que permite controlar a variabilidade das características dos participantes (Fowler, 1993), embora torne, muitas vezes, os projetos de pesquisa descritiva de difícil operacionalização devido a custos ou ao tempo de coleta de dados.

Os dados-chave para a realização de pesquisas descritivas são os levantamentos, frequentemente realizados por meio de survey, mas outros tipos de dados podem ser utilizados de modo complementar, como bancos de dados ou pesquisa qualitativa. Cabe ressaltar que nenhum dos outros dados por si só é capaz de substituir os de levantamentos, já que seus interesses não incluem a descrição de dada realidade de mercado de forma representativa (Aaker et al., 2004; Malhotra \& Peterson, 2001). Perosa, Tarsitano, Martins, Pigatto e Antonangelo (2012) descreveram o perfil de consumidores de frutas do interior do Estado de São Paulo. Utilizando uma amostra de 404 pessoas, distribuídas em diversas cidades, foi possível descrever aspectos como a preocupação das pessoas com características do produto (saudáveis e saborosos), preço e acesso aos produtos.

Nos surveys, podem-se utilizar questões abertas ou fechadas, incluindo o uso de escalas diversas para mensuração das respostas (ex., Nepomuceno \& Torres, 2005). Diferentemente do que ocorre na pesquisa exploratória, o pesquisador não é elementochave para a compreensão dos fenômenos estudados. Ao contrário, há a tentativa de neutralizar influências que não sejam provenientes do próprio consumidor (Aaker et al., 2004; Malhotra \& Peterson, 2001).

Apesar de extremamente eficaz para a medição de uma série de variáveis relacionadas ao consumo, a pesquisa descritiva apresenta um espaço limitado para a descoberta de relações entre as variáveis pesquisadas. Tomando os surveys como exemplo, os insights obtidos por meio do processo de pesquisa não ultrapassarão as possibilidades de cruzamento de resultados de questões integrantes do instrumento. Pelo contrário, nas técnicas estruturadas de coleta de dados, o que será investigado já está definido quando se vai a campo. O contrário ocorre na pesquisa exploratória, que, por ser menos estruturada, tem maior possibilidade de descoberta de dados inesperados e valiosos, podendo até mesmo suscitar novas ideias essencialmente diferentes das esperadas no princípio da pesquisa, como, por exemplo, a possibilidade de desenvolvimento de um novo conceito de produto inexistente no mercado.

Fabris, Steiner Neto e Toaldo (2010) desenvolveram um estudo que utilizou ambas as abordagens referidas - exploratória e descritiva - a fim de avaliar, entre universitários, a influência dos agentes de socialização (família, escola, mídia e pares) em relação aos hábitos de reciclagem, ao conhecimento sobre o assunto e aos sentimentos relacionados ao tema. A pesquisa, realizada com 351 pessoas, teve duas etapas, sendo a primeira exploratória, e a segunda, descritiva. Os resultados mostram que os quatro agentes influenciam o comportamento das pessoas, sendo 
possível, com a contribuição de ambos os métodos, compreender tanto fatores mais influentes (etapa descritiva) quanto o processo de concretização da influência (etapa exploratória).

Mesmo levando-se em consideração que os processos de pesquisa descritiva são mais estruturados, deve-se atentar para o desenvolvimento dos instrumentos de pesquisa, procurando alternativas que assegurem que os dados inseridos nas questões do survey não sejam impostos pelos pesquisadores, mas condizentes com a realidade do mercado. Para tanto, é comum que se utilize uma etapa exploratória para levantar questões e alternativas de respostas a serem contempladas no instrumento de coleta de dados da pesquisa (Aaker et al., 2004; Malhotra \& Peterson, 2001).

Apesar de o uso da internet ser uma tendência em pesquisa de mercado (Malhotra \& Peterson, 2001), sua utilização pode comprometer o potencial de generalização dos dados para a população geral. Isso se deve às diferenças do perfil dos respondentes que participam das pesquisas pela internet, já que essas pessoas tendem a ser usuários mais frequentes do meio e, portanto, compartilhariam características que destoam da população geral. Os estudos que têm a internet como fonte de dados devem, preferencialmente, restringir-se à investigação de informações relacionadas a produtos ou a serviços comuns a usuários desse meio.

Como alternativa, o uso do telefone para a realização de pesquisas de survey ainda é boa opção para alcançar uma população mais ampla, sendo dominante nos EUA, no Canadá e em alguns países europeus, como a Suécia. Já na França e na Holanda, predominam as entrevistas nas ruas como a técnica mais difundida para a coleta de dados (Malhotra \& Peterson, 2001). No Brasil, uma revisão sobre a pesquisa e a produção científica do início do século XXI destaca que os estudos cada vez mais se utilizam de formas estruturadas de pesquisas de survey (Sampaio \& Perin, 2006).

Os objetivos das pesquisas descritivas, portanto, não buscam entender o processo de como se dá um problema (por exemplo, entender como o consumidor italiano se relaciona com produtos geneticamente modificados), como nas pesquisas exploratórias, mas visam a possibilitar um retrato da realidade a fim de que se possa fazer um mapeamento de comportamentos. Da mesma forma, a pesquisa descritiva pode mapear a realidade, mas não é usualmente aplicada para inferência de nexos causais (por exemplo, investigando o impacto da variação de preços no volume de vendas entre alimentos e produtos de limpeza [OliveiraCastro, 2003]), como a pesquisa experimental (para uma visão mais geral, ver Tabela 1).

Se o que interessa é justamente obter uma imagem da realidade para conhecer o potencial de mercado para o lançamento de um novo produto, deve-se assegurar que os dados da pesquisa sejam os mais próximos possível do que ocorre no cotidiano do consumidor e, portanto, desenvolver uma pesquisa descritiva (Aaker et al., 2004; Malhotra \& Peterson, 2001). Trata-se de um tipo de pesquisa que convém justamente ao suporte das decisões nas empresas, pois é o método com maior potencial para realizar descrições sobre o mercado.

Etapas de pesquisa descritiva são comumente incorporadas a projetos que envolvem outras abordagens como métodos principais. Um estudo para o desenvolvimento de um conceito para um produto que preveja uma pesquisa exploratória pode exigir, em uma etapa inicial, um estudo descritivo que ofereça um mapeamento das características do público-alvo. Um estudo experimental no qual se deseja testar o impacto de modificações em um produto, como cores 
de embalagem, sabores e quantidades (variáveis independentes), sobre o volume de vendas (variável dependente) pode exigir, em etapa introdutória, um estudo descritivo para levantar os principais motivos de descarte do produto em relação a alternativas dos concorrentes. Entretanto, conforme salientado, apesar de seu potencial para a realização de generalizações a partir dos resultados, esse tipo de pesquisa não proporciona a investigação de relações de causa e efeito, conforme será explorado na seção seguinte.

\section{O uso de experimentos no estudo do comportamento do consumidor}

Como é possível afirmar que um fenômeno é consequência de uma ou de mais causas? A pesquisa experimental é o tipo de pesquisa mais indicado para traçar relações de causa e efeito, em geral lançando mão, para tanto, do uso de métodos quantitativos e de análises estatísticas. Experimentos são estudos nos quais as condições devem ser controladas a fim de possibilitar a avaliação independente da manipulação de variáveis (Aaker et al., 2004). O pesquisador manipula (ou seja, modifica) uma ou mais variáveis, como o meio de divulgação de produtos, as quais chama de variáveis independentes ou experimentais, no intuito de averiguar se essas têm efeito sobre outro tipo de variável, como motivação para compra, chamada de variável dependente.

Desse modo, manipulando a variável em estudo (ex., apresentar um produto em revista, em outdoors ou na televisão), o pesquisador visa a mensurar o efeito sobre a variável dependente (aumento da procura pelo produto no mercado). Para análises de dados quantitativos, em geral os pesquisadores lançam mão de diversos softwares estatísticos. A pesquisa experimental possibilita, nesse sentido, que sejam respondidas questões como: Quais os efeitos do aumento de preços sobre as vendas de dado produto? A quantidade de visitas dos vendedores aos consumidores modifica o número de pedidos? (Humphrey, Ellis, Conlon, \& Tinsley, 2004).

Para comparar se uma resposta muda em função da manipulação do estímulo, frequentemente utilizam-se pelo menos dois grupos. Um deles é denominado grupocontrole, e a ele não se apresenta a variável independente manipulada, enquanto o outro é chamado de grupo experimental, já que a ele se apresenta a variável independente manipulada ou modificada. Tonetto, Rohenkohl e Stein (2008) estudaram o efeito da pressão do tempo (i.e., da definição de prazos) na tomada de decisão do consumidor. Para tanto, foi desenvolvido um software que apresentou doze dilemas a consumidores universitários distribuídos em três grupos. $\mathrm{O}$ primeiro grupo, o grupo-controle, respondeu aos problemas com tempo livre, enquanto o segundo e o terceiro grupos, os grupos experimentais, tiveram o tempo-limite reduzido para responder a cada dilema. Essa redução foi calculada com base no tempo despendido pelos integrantes do grupocontrole para responderem a cada questão. Os resultados permitiram aos autores afirmar que a definição de prazos para a tomada de decisão pode modificar as respostas dos participantes.

Em outro exemplo, com o objetivo de avaliar o impacto da quantidade de informações e da configuração da informação (forma como é apresentada, se ressaltando ganhos ou perdas das mesmas situações de consumo) sobre o grau de persuasão de uma mensagem de comunicação sobre um produto de crédito, Tonetto e Stein (2012) realizaram um estudo experimental. Participaram do experimento quatro grupos de consumidores constituídos em função das variáveis configuração da 
informação (ganhos e perdas) e quantidade de informações (expandidas e resumidas). Um efeito de configuração das mensagens foi detectado apenas entre as versões resumidas do texto, sendo a configuração de ganhos considerada a mais convincente.

Os estudos experimentais de mercado apresentam importantes limitações, pois os ambientes ideais para sua realização são artificiais, controlando as mesmas condições do local da testagem para todos os participantes. A realidade do mercado, no entanto, faz com que o pesquisador precise abrir mão do rigor do controle de variáveis experimentais, o que ameaça a validade do estudo, pois, dessa forma, a variável dependente pode sofrer interferência de outras variáveis não controladas, além daquela que foi deliberadamente manipulada.

Como exemplo dessa interferência, pode-se citar a pesquisa sobre uma redução de preços para a avaliação de seu impacto sobre o volume de vendas. Estas podem variar tanto em função da variação dos novos preços em teste quanto em função da proximidade do Natal, mas, devido à ineficácia do controle total do ambiente no qual o estudo estaria em realização (como ocorre com maior facilidade nos ambientes artificiais, ou laboratórios), não se pode afirmar as reais causas de possíveis modificações no volume de vendas, uma vez que não há possibilidade de controlar todas as variáveis envolvidas no ambiente natural.

Luppe e Angelo (2010), visando a mensurar os efeitos da ancoragem (sugestão de um valor arbitrário) na percepção e na avaliação de preços de produtos e serviços, conduziram um experimento com universitários em um ambiente artificial (aula), contando com três grupos. O grupo-controle estimou o preço de produtos e serviços sem menção a âncoras, enquanto os dois grupos experimentais estimaram os valores frente a âncoras. Os resultados revelaram que os referenciais com valores menores tiveram maior efeito sobre as percepções de preços, pois ancoram mais fortemente as avaliações realizadas.

Alternativas em relação ao uso de laboratórios estão em constante investigação. Assim como os métodos informatizados dos estudos de Tonetto et al. (2008), a internet é um veículo bastante utilizado para conduzir pesquisas experimentais. Os integrantes de uma amostra podem ser recrutados para responder, em sites, questões relacionadas à variável dependente. Assim, a internet provê um mecanismo para experimentação diverso dos laboratórios (Malhotra \& Peterson, 2001).

Diferentemente da pesquisa exploratória, a interferência do pesquisador nos experimentos não é desejada (para comparação, ver Tabela 1). Qualquer possível causa de modificação nos efeitos entre as variáveis deve ser eliminada (Humphrey et al., 2004). Nos experimentos de Tonetto et al. (2008) citados anteriormente, todos os integrantes da amostra receberam informações equivalentes sobre o tema da pesquisa, para assegurar que o experimentador não exerceria influência distinta sobre os grupos. Além disso, como uma forma de assegurar que os estímulos apresentados pelo software fossem equivalentes para os três grupos (ex., cronômetro na tela), os integrantes do grupocontrole visualizaram, na tela do computador, um cronômetro progressivo, enquanto aos integrantes dos grupos experimentais foi apresentado um cronômetro regressivo.

Estudos exploratórios podem auxiliar a definir um experimento. A fim de investigar a relação entre a presença de aroma em lojas e as avaliações de consumidores sobre a loja, o ambiente, os produtos, as intenções de retorno e o tempo gasto na loja, Costa e Farias (2011) realizaram um estudo exploratório anterior ao experimento a fim de delinear as variáveis. Os resultados mostraram que o aroma ambiental não melhorou as avaliações 
dos consumidores e a intenção de retorno à loja, mas reteve o consumidor por mais tempo no ambiente.

A amostra deve ser cuidadosamente selecionada, respeitando-se os critérios de aleatoriedade para que os dados possam ser confiáveis e representativos para a população pesquisada. Os dados de relatórios experimentais, portanto, pretendem ser conclusivos em relação ao fato de uma variável (independente) causar modificações em outra (dependente), predizendo como a variável dependente se comportará frente à independente. Como exemplo de bom procedimento de seleção da amostra, cita-se a pesquisa realizada pela empresa Campbell Soup, na qual foi realizada uma série de experimentos em ambiente natural para avaliar alternativas de estratégia publicitária (Aaker et al., 2004). Um dos experimentos avaliou a mudança de $25 \%$ de verba de TV para outdoors em dois mercadosteste (Houston e Indianápolis), enquanto, nos demais mercados, mantiveram-se os investimentos-padrão em mídias. As vendas foram mensuradas a cada quatro semanas durante o experimento e nos três anos anteriores. Foi verificado um aumento de $8 \%$ nas vendas em oito meses nos mercados-teste, devido ao fato de os outdoors terem atingido um público que não era atingido pela TV.

Uma questão interessante se refere ao fato de a Campbell Soup utilizar as vendas de três anos anteriores como comparativo. Esse tipo de informação serve como base para que se possa realmente afirmar que foi a mudança de mídia a responsável pelo aumento das vendas, e não mudanças situacionais, promoções ou tendências de consumo ao longo dos tempos.

O uso de experimentos também pode ser importante em etapas iniciais de um projeto exploratório ou descritivo, com a finalidade de auxiliar a melhor definir o problema a ser pesquisado. Uma pesquisa exploratória também pode ser motivada por resultados de pesquisa experimental. Um experimento que vise a investigar a percepção do consumidor gaúcho sobre o sabor (variável dependente) de ervas de chimarrão com pequenas quantidades de chá pode manipular uma determinada marca de erva com diferentes tipos de chá (variável independente). Caso o resultado desse estudo indicasse que as diferentes configurações de ervas não mostraram diferentes percepções do públicoalvo, esse resultado apontaria a necessidade de uma pesquisa exploratória que avaliasse a real relação entre o consumidor e a erva de chimarrão, já que o motivo de consumo sugeriria não ser a inserção do chá na erva. Os dados advindos do resultado dessa pesquisa podem gerar uma nova pesquisa experimental.

O uso de pesquisa experimental também pode anteceder estudos descritivos, que avaliem, por exemplo, a disposição de um determinado público-alvo para a aquisição de um novo produto. $\mathrm{O}$ foco da pesquisa experimental poderia ser o teste do efeito de diferentes sabores, cores e embalagens, entre outros (variáveis independentes), na maior probabilidade de aquisição do produto (variável dependente) antes mesmo de se testar o potencial de mercado.

A pesquisa experimental constitui, dessa forma, método para traçar relações de causa e efeito. A pesquisa descritiva, apesar de utilizar dados quantitativos, apenas descreve a realidade, de modo que não é possível ter noção da causa dos fenômenos em estudo. A pesquisa exploratória, por outro lado, pode investigar os motivos da associação de variáveis, mas não afirmar a existência de graus de relações entre elas. A condução de experimentos, nessa direção, proporciona uma fonte segura de informações sobre o comportamento dos consumidores, possibilitando afirmações eficazes no que se refere a relações de causalidade entre variáveis (ver Tabela 1 para um panorama). 
A escolha do método, em estudos orientados para o mercado, é determinada pelo problema de pesquisa, não pelo

pesquisador, pela sua orientação teórica ou pelo seu grau de conhecimento (Gilgun, 2005).

\section{Considerações finais: uma visão integradora sobre os diferentes métodos de pesquisa}

Cada tipo de pesquisa - exploratória, descritiva e experimental - tem indicações particulares no que se refere ao potencial explicativo dos resultados alcançados, de modo que não há terreno para a disputa entre métodos no sentido de definir qual o mais completo ou útil. O desafio, portanto, consiste em identificar qual o método de pesquisa mais indicado para cada tipo de pesquisa. A escolha do método, em estudos orientados para o mercado, é determinada pelo problema de pesquisa, não pelo pesquisador, pela sua orientação teórica ou pelo seu grau de conhecimento (Gilgun, 2005).

A atuação do pesquisador, nessa direção, exige formação metodológica generalista, ou seja, conhecimento sobre diferentes tipos de pesquisa. Quando não se tem o domínio sobre o método mais indicado, a utilização de outro método poderá acarretar sérios problemas para a empresa contratante no que tange à tomada de decisão em marketing, pois os dados podem não ser seguros para gerar as respostas necessárias para a empresa. Em síntese, cada método tem peculiaridades que fazem com que todos eles sejam igualmente úteis em contextos distintos (ver Tabela 1 para complemento).

O novo milênio trouxe a tendência de integração nos métodos de pesquisa para responder a um cenário de mercado em constantes transformações. Na prática em pesquisa, o profissional se depara com situações nas quais o emprego complementar dos três tipos de pesquisa descritas pode ser interessante, mas em momentos distintos (Gilgun, 2005). Nesses casos, a integração de métodos pode ser a opção mais adequada, uma vez que permite que as limitações de um método sejam compensadas pelas vantagens da utilização de outro (Iglesias \& Alfinito, 2006).

A fim de exemplificar uma abordagem integradora entre os diferentes métodos e comparar as propriedades de cada um, serão utilizados dois exemplos de pesquisa, o primeiro sobre o lançamento de uma nova marca de sorvetes, e o segundo, sobre planos de vida de jovens que ingressam no ensino superior em dada universidade privada.

(a) Lançamento de uma nova marca de sorvetes

Imagine que uma pesquisa foi solicitada para o lançamento de uma nova marca de sorvetes, que, nesse exemplo, integraria uma categoria intermediária entre as marcas populares e as Premium existentes no cenário nacional. O pesquisador, nesse caso, deveria elencar elementos que integrariam um conceito para essa marca e, ainda, a escolha de sabores dos produtos.

O processo de pesquisa poderia iniciar com um estudo descritivo que avaliasse o potencial de mercado para a nova marca, oferecendo, ao gestor, dados que apontem a real parcela do mercado disposta a consumir seu lançamento. Para isso, a perspectiva da pesquisa descritiva poderia considerar as diferentes praças envolvidas, as marcas existentes no mercado, as características do consumidor em si, os preços praticados e as promoções, entre outros, avaliando todas essas dimensões por meio da utilização de survey.

Supondo que exista espaço no mercado para o lançamento da referida marca (detectado por meio da etapa descritiva), uma pesquisa exploratória, realizada por meio de entrevistas em profundidade ou de grupos focais, poderia ser utilizada para o desenvolvimento de um conceito para o produto, exaltando, por exemplo, a relação custo-benefício, caso o argumento-chave identificado na 
pesquisa exploratória fosse esse. Assim, dados provenientes do consumidor auxiliariam no desenvolvimento de um conceito que efetivamente fosse absorvido pelas pessoas.

Por fim, a avaliação de quais sabores (variável independente) implicariam melhor avaliação do sabor do sorvete (variável dependente) poderia ser desenvolvida por meio de uma pesquisa experimental realizada com o público-alvo da nova marca. A utilização de experimentos possibilita ainda testar o impacto de diferentes aromas, texturas, embalagens ou cores (variáveis independentes) sobre o consumo do produto ou sobre sua avaliação (variáveis dependentes).

Nesse exemplo, a integração de diferentes métodos de pesquisa em um mesmo projeto seria essencial para responder à demanda da empresa. O uso de um método único não seria, nessa perspectiva, suficiente para compreender a realidade do mercado em questão.

(b) Planos de vida de jovens que ingressam no ensino superior em dada universidade privada

Imagine agora que um projeto foi solicitado para compreender como são construídos os planos de vida de jovens que ingressam no ensino superior em dada universidade privada, apontando a articulação desses planos com a realização de um curso superior. O estudo deveria contemplar a investigação do impacto do uso de palestras educativas em escolas como forma de prospectar novos estudantes.

Uma pesquisa do tipo exploratória poderia tratar da investigação sobre como os jovens constroem seus projetos de vida e que relações esses planos têm com o ingresso no ensino superior. Para tanto, mais uma vez, o uso de técnicas de coleta de dados como entrevistas em profundidade ou grupos focais seria de primeira escolha. Essa etapa de pesquisa poderia demonstrar a forma como tais projetos de vida são construídos, identificando os fatores associados a essa construção, o que possibilitaria insights sobre o fenômeno em estudo.

A pesquisa descritiva poderia ser desenvolvida a seguir, utilizando-se o survey, visando ao mapeamento de frequência de tipos de planos de vida mais comuns e suas articulações com o ensino superior. Dessa forma, seria possível identificar, inclusive, lacunas no mercado para o desenvolvimento de novos cursos de graduação.

Experimentos avaliariam o impacto de palestras educativas nas escolas da região (variável independente) na procura pela universidade e o ingresso no ensino superior (variáveis dependentes). Seria possível, ainda, desenvolver experimentos que salientassem, na comunicação da universidade, os elementos relacionados aos diferentes planos de vida dos estudantes (variável independente) no acesso ao site da universidade e no número de inscrições no vestibular (variáveis independentes), por exemplo.

Mais uma vez, o uso dos diferentes métodos mostra-se essencial no sentido de oportunizar a compreensão dos fenômenos em estudo. Conhecer os diferentes tipos de pesquisa e abordagens distintas em função da demanda das empresas parece suprir os desafios metodológicos apontados no presente artigo. Essa necessidade decorre do fato de o comportamento do consumidor ser um fenômeno complexo, que pode requerer a condução de estudos exploratórios (encontrados usualmente em estudos culturais, de influências de grupos e de valores humanos), descritivos (comuns em pesquisas de intenção, de opinião e em mapeamentos de potencial de mercado) ou experimentais (frequentes em estudos relacionados ao impacto de variáveis como variações nos produtos, na distribuição, na promoção ou 
em preços sobre as decisões de compra). $\mathrm{O}$ mercado contemporâneo necessita, assim, de pesquisadores preparados para responder a diferentes demandas.

Nessa visão, ratifica-se que o que define a escolha do método é o foco da pesquisa. Existem métodos adequados a cada tipo de problema, restando ao pesquisador a tarefa de conhecê-los e de saber indicálos quando preciso. A pesquisa sobre o comportamento do consumidor chegou, portanto, a níveis de conhecimento e de diferenciação metodológica altamente especializados. O pesquisador, além de gestor de marketing, necessita conhecer a diversidade metodológica que o campo apresenta. Isso significa que, além de compreender as mudanças do mercado, necessita saber pesquisar os consumidores e integrar essas informações ao planejamento e à gestão de marketing, o que constitui um desafio importante em termos de formação de psicólogos. 


\section{Leandro Miletto Tonetto}

Doutor em Psicologia (Área de Cognição Humana) pela Pontifícia Universidade Católica do Rio Grande do Sul e professor do Programa de Pós-graduação em Design da Universidade do Vale do Rio dos Sinos, Porto Alegre - RS - Brasil.

E-mail: Itonetto@gmail.com

\section{Priscila Goergen Brust-Renck}

Doutoranda em Developmental Psychology no Departamento de Human Development na Cornell University nos Estados Unidos, Ithaca - Estados Unidos.

E-mail: prirenck@gmail.com

\section{Lilian Milnitsky Stein}

Doutora em Cognitive Psychology pela University of Arizona e Professora titular do Programa de Pós-Graduação em Psicologia da Pontifícia Universidade Católica do Rio Grande do Sul, Porto Alegre - RS - Brasil.

E-mail: lilian@pucrs.br

\section{Endereço para envio de correspondência:}

Rua Luiz Manoel Gonzaga, 744. CEP: 90470-280. Porto Alegre, RS.

Recebido 19/03/2012, 1a Reformulação 06/05/2013, Aprovado 29/08/2013.

\section{Referências}

Aaker, A. D., Kumar, V., \& Day, G. S. (2004). Marketing research (8a ed.). New York: John Wiley \& Sons.

Abdullah, F. (2006). Measuring service quality in higher education: HEdPERF versus SERVPERF. Marketing Intelligence \& Planning, 24(1), 31-47. doi/pdf/10.1086/429604doi/ pdf/10.1086/429604

Barboza, M. N. L., \& Arruda Filho, E. J. M. (2012). O comportamento do consumidor tecnológico diante dos valores ecologicamente corretos: ideologia verde versus responsabilidade social. Intercom - RBCC, 35(1), 157-182. doi: http://dx.doi.org/10.1590/S1809-58442012000100009

Canniford, R. (2005). Moving shadows: Suggestions for ethnography in globalized cultures. Qualitative Market Research: An International Journal, 8(2), 204-218. doi: 10.1108/13522750510592463
Costa, A. L. C. N., \& Farias, S. A. (2011). O aroma ambiental e sua relação com as avaliações e intenções do consumidor no varejo. Revista de Administração de Empresas, 51(6), 528-541.

Coupland, J. C. (2005). Invisible brands: An ethnography of households and the brands in their kitchen pantries. Journal of Consumer Research, 32(1), 106-118. doi:

Erasmus, A., Boshoff, E., \& Rousseau, G. (2001). Consumer decision-making models within the discipline of consumer science: A critical approach. Journal of Family Ecology and Consumer Sciences, 29, 82-90. Doi:

Fabris, C., Steiner Neto, P. J., \& Toaldo, A. M. (2010). Evidências empíricas da influência da família, mídia, escola e pares nos antecedentes e no comportamento de separação de materiais para a reciclagem. Revista de Administração Contemporânea, 14(6), 1134-1157. 
Fontes, O. A., Borelli, F. C., \& Casotti, L. M. (2012). Como ser homem e ser belo? Um estudo exploratório sobre a relação entre masculinidade e o consumo de beleza. REAd, 72(2), 400-432.

Foxall, G. R. (2001). Foundations of consumer behaviour analysis. Marketing Theory, 1(2), 165-199. doi: 10.1177/147059310100100202

Foxall, G. R. (2003). The behavior analysis of consumer choice: An introduction to the special issue. Journal of Economic Psychology, 24(5), 581-588. doi: http://dx.doi.org/10.1016/ S0167-4870(03)00002-3

Fowler, F. J., Jr. (1993). Applied social research methods series: Survey research methods (Vol. 1, 2a ed.). Newbury Park: SAGE.

Gilgun, J. F. (2005). Evidence-based practice, descriptive research and the resilience-schema-gender-brain functioning (RSGB) Assessment. British Journal of Social Work, 35(6), 843-862.

Gouvêa, M. A., Oliveira, B., \& Nakagawa, S. S. Y. (2013). Lealdade em compras online versus offline: reflexões sobre os fatores relevantes. Organizações \& Sociedade, 20(64), 37-53. doi: http://dx.doi.org/10.1590/S1984-92302013000100004

Hassan, S., Craft, S., \& Kortam, W. (2003). Understanding the new bases for global market segmentation. Journal of Consumer Marketing, 20(5), 446-462. doi: 10.1108/07363760310489670

Humphrey, S. E., Ellis, A. P. J., Conlon, D. E., \& Tinsley, C. H. (2004). Understanding customer reactions to brokered ultimatums: Applying negotiation and justice theory. Journal of Applied Psychology, 89(3), 466482. doi: 10.1037/0021-9010.89.3.466

Iglesias, F., \& Alfinito, S. (2006). A abordagem multimetodológica em comportamento do consumidor: dois programas de pesquisa na oferta de serviços. Psicologia: Organizações e Trabalho, 6(1), 138-165.

Livramento, M. N., Hor-Meyll, L. F., \& Pessôa, L. A. G. (2013). Valores que motivam mulheres de baixa renda a comprar produtos de beleza. Revista de Administração Mackenzie, 14(1), 44-74. doi: http://dx.doi.org/10.1590/S167869712013000100003

Luppe, M. R., \& Angelo, C. F. (2010). As decisões de consumo e a heurística da ancoragem: uma análise da racionalidade do processo de escolha. RAM, Revista de Administração Mackenzie (online), 11(6), 81-106.doi: http://dx.doi. org/10.1590/S1678-69712010000600006

Malhotra, N. K. (2006). Pesquisa de marketing: uma orientação aplicada (4a ed.). Porto Alegre: Bookman.

Malhotra, N., \& Peterson, M. (2001). Marketing research in the new millennium: Emerging issues and trends. Marketing Intelligence \& Planning, 19(4), 216-235. doi: 10.1108/ EUM0000000005560

Nepomuceno, M. V., \& Torres, C. V. (2005). Validação da Escala de Julgamento e Significado do Produto. Estudos de Psicologia (Natal), 10(3), 12-28. doi: http://dx.doi.
org/10.1590/S1413-294X2005000300011

Nepomuceno, M. V., Porto, R. B., \& Rodrigues, H. (2006). Avanços teóricos e metodológicos das pesquisas sobre julgamento e significado do produto no Brasil. Psicologia: Organizações e Trabalho, 6(1), 106-137.

O'Connor, H., \& Madge, C. (2003). "Focus groups in cyberspace": Using the internet for qualitative research. Qualitative Market Research: An International Journal, 6(2), 133-143. doi: 10.1108/13522750310470190

Oliveira, B., \& Gouvêa, M. A. (2010). A importância das ações sociais empresariais nas decisões de compra dos consumidores. Gestão da Produção, 17(4), 791-800. doi: http://dx.doi.org/10.1590/S0104-530X2010000400012

Oliveira-Castro, J. M. (2003). Effects of base price upon search behavior of consumers in a supermarket: An operant analysis. Journal of Economic Psychology, 24, 637-652. doi: http:// dx.doi.org/10.1016/S0167-4870(03)00006-0

Perosa, J. M. Y., Tarsitano, M. A. A., Martins, M. I. E. G., Pigatto, G., \& Antonangelo, A. (2012). Perfil do consumidor de frutas em cidades do interior do Estado de São Paulo. Revista Brasileira de Fruticultura, 34(4), 1084-1090. doi: http://dx.doi. org/10.1590/S0100-29452012000400015

Sampaio, C. H., \& Perin, M. G. (2006). Pesquisa científica na área de marketing: uma revisão histórica. Revista de Administração Contemporânea, 10(2), 179-202. doi: http:// dx.doi.org/10.1590/S1415-65552006000200010

Secchi, J, Silva, W. V., Del Corso, J. M., \& Tortato, U. (2012). Relações de gênero no comportamento de compra pela internet: estudo das dimensões do risco percebido. Sociais e Humanas, 25(2), 253-272.

Simonson, I., Carmon, Z., Dhar, R., Drolet, A., \& Nowlis, S. M (2001). Consumer research: In search of identity. Annual Review of Psychology, 52(1), 249-275. doi: 10.1146/annurev. psych.52.1.249

Tonetto, L. M., Rohenkohl, G., \& Stein, L. M. (2008). O efeito da pressão do tempo na tomada de decisão do consumidor. Revista Interamericana de Psicologia, 42(1), 119-128.

Tonetto, L. M., \& Stein, L. M. (2012). Psicologia cognitiva e consumo: o impacto da quantidade de informações na configuração de objetivos. Psicologia: Reflexão e Crítica, 25(1), 174-181. doi: http://dx.doi.org/10.1590/S010279722012000100021

Torres, C. V., Allen, M. W., \& Pérez-Nebra, A. R. (2006). Consumo no Brasil: um teste etic da relação entre valores humanos, significado do produto e preferência de consumo em culturas coletivas. Psicologia: Organizações e Trabalho, 6(1), 79-105

Vieira, V. A., \& Tibola, F. (2005). Pesquisa qualitativa em marketing e suas variações: trilhas para pesquisas futuras. Revista de Administração Contemporânea, 9(2), 9-33. doi: http://dx.doi. org/10.1590/S1415-65552005000200002 